\title{
DEVELOPMENT OF MODELS OF SPATIAL ANALYSIS OF STATUS OF INTERACTIVE PROCESSES OF COMPLEX SYSTEMS
}

\section{Tvoroshenko Iryna ${ }^{1}$}

DOI: https://doi.org/10.30525/978-9934-571-89-3_121

Given that objects of special purpose function in a priori uncertainty, characterized by a fuzzy space of states, which requires new intellectual approaches to increase the reliability of the decisions that are adopted, characterized by functional and territorial distribution, a complex hierarchy of interacting processes [1]. It is necessary to predict certain requirements to the mathematical apparatus, methods of objectoriented modeling and analysis of interacting processes of a complex system.

Existing approaches to analyzing, modeling and constructing complex data management and processing systems under these conditions are ineffective because of their functional limitations. It should be noted that the promising apparatus for constructing complex systems is the use of fuzzy logic by Lotfi Zade, and for the analysis and modeling of interacting processes of complex systems. It is expedient to use the petri net system (PN) [1] and its extensions [2].

Given the significant limitations and assumptions, as well as the drawbacks of known models, new solutions and approaches to the further development of ideas for the construction of mathematical models are proposed [2]. Classes of fuzzy network models (FNM) are considered which integrate the advantages of models based on PN and neuro-fuzzy networks (NFN).

Mathematical interpretation of FNM is given as:

$$
\tilde{S}(f)=<\tilde{P}, \tilde{T}, \tilde{F}(f), \tilde{M}(f)_{0}, L>,
$$

where $\widetilde{P}=\left\{\tilde{p}_{j}: \mu_{\widetilde{p}_{j}}(k)\right\}-$ the finite set of fuzzy positions $\tilde{p}_{j}$;

${ }^{1}$ Kharkiv National University of Radio Electronics, Ukraine 
$\mu_{\widetilde{p}_{j}}(k)$ - the function of belonging to the $j$-th fuzzy set position $\tilde{P}$;

$k$ - some variable that defines the function argument

$$
\mu_{\tilde{p}_{j}}(k), \quad j=\overline{1, m}, \quad \widetilde{P} \neq \varnothing, \quad|\widetilde{P}|=m ;
$$

$\widetilde{T}=\left\{\widetilde{t}_{i}: \mu_{\tilde{t}_{i}}(k)\right\}$ - the finite set of fuzzy transitions $\tilde{t}_{i}, i=\overline{1, n}, \quad \widetilde{T} \neq \varnothing,|\widetilde{T}|=n$; $\mu_{\tilde{t}_{i}}(k)$ - the function of belonging to the $i$-th fuzzy transition;

$$
\widetilde{F}(f):(\tilde{P} \times \tilde{T}) \cup(\tilde{T} \times \widetilde{P}) \rightarrow\left\{x_{i j}(k), y_{i j}(k)\right\} .
$$

$\widetilde{F}(f)$ - fuzzy function of incidence $\widetilde{P}$ and $\widetilde{T}$;

$x_{i j}(k), y_{i j}(k)$ - Functionalities of incoming and outgoing incidents of some fuzzy positions $\tilde{p}_{j} \in \widetilde{P}$ and fuzzy transitions $\tilde{t}_{i} \in \tilde{T}$;

It should be noted that the initial fuzzy space of states of the model (1) is determined by the vector of fuzzy initial marking $\tilde{M}(f)_{0}$ of fuzzy model positions $\widetilde{P}$ :

$$
\tilde{M}(f)_{0}=\left\{\tilde{M}\left(\tilde{p}_{j}\right): z_{\tilde{p}_{j}}(k),\right.
$$

$\tilde{M}\left(\tilde{p}_{j}\right) \rightarrow[0,1]-$ fuzzy marking of a fuzzy position $\tilde{p}_{j} \in \widetilde{P}$ FNM;

$z_{\tilde{p}_{j}}(k)$ - the function of the marking of the $j$-th fuzzy position $\tilde{p}_{j} \in \widetilde{P}$;

$L$ - some predicate, which depends on the set of variables $\left\{x_{u}\right\}, u \in U$.

During the study, it was determined that with the increase in the dimension of a complex system in FNM it is necessary to take into account $\left\{x_{u}\right\}, u \in U-\mathrm{a}$ set of additional parameters, characteristics and conditions. It is established that an effective mechanism for reducing the dimension of FNM (1) can be considered colored fuzzy Petri networks (CFPN).

In [2], the concept of CFPN introduced in this way is introduced:

$$
\tilde{S}_{C}(f)=<\tilde{P}, \tilde{T}, \tilde{F}_{C}(f), \tilde{M}_{O C}(f), \tilde{M}_{C}(f), L\left\{x_{u}\right\}, \tilde{C}, \tilde{V}, \tilde{K}>,
$$

where $\widetilde{P}-$ set of fuzzy positions;

$\widetilde{T}-$ set of fuzzy transitions;

$$
\widetilde{F}_{C}(f)=(\tilde{P} \times \tilde{T}) \cup(\tilde{T} \times \widetilde{P}) .
$$

$\widetilde{F}_{C}(f)$ - fuzzy function of network incidents $\widetilde{S}_{C}(f)$;

$\tilde{M}_{C}(f)_{0}$ - vector of initial marking;

$\tilde{M}_{C}(f)$ - vector of current marking;

$L\left\{x_{u}\right\}, u \in U-$ a certain predicate, assigned to the set of positions, transitions, functions of incidence in the space of states of fuzzy interacting processes, which determines the additional conditions for the implementation of transitions; 
$\widetilde{C}$ - a marker color function that defines the color of each marker $\tilde{M}\left(\tilde{p}_{j}\right)$ for the network position;

$\tilde{V}$ - conditions for the operation of transitions depending on the color of the marker;

$\widetilde{K}$ - number of markers in positions with the account $\widetilde{C}$.

Consequently, as follows from (4), the network integrates the network (3) and the advantages of CFPN. In addition, the introduction of the predicate $L\left\{x_{u}\right\}, u \in U$ into model (4), as well as properties $\tilde{C}, \tilde{V}, \tilde{K}$ significantly increases the possibilities of the model compared with existing approaches.

Thus, it can be argued that (4) is a significant extension of FNM.

Studies have shown that this approach is effective in practical implementation and reduces the dimensionality of the investigated processes.

Analyze the test case, when the process can be run only if several conditions are fulfilled, and consider the additional capabilities of CFPN (4) as compared to the FNM (1). Let, for certainty, there is a process $\tilde{d}_{r}$, for which it is necessary to fulfill three conditions: $\widetilde{U}_{1}, \widetilde{U}_{2}, \widetilde{U}_{3}$ from a plurality $\widetilde{U}_{l}, l \in L$.

We construct a fragment of the model of interacting processes using fuzzy network models (1). The conditions of the three positions $p_{1}, p_{2}, p_{3}$ (Fig. 1a) ensure the implementation of the transition $t_{l}$ (the process is carried out $\tilde{d}_{r}$ ).

In Figure $1 \mathrm{~b}$ we will demonstrate this fragment of the model using painted fuzzy Petri nets (4). In Figure 1b, the position $p_{3}$ is marked with three-color labels, which is conventionally shown as an additional marking of some conventional positions $p_{1}, p_{2}$.

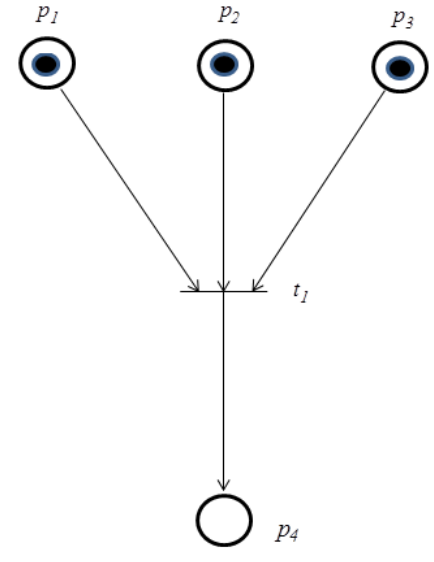

a

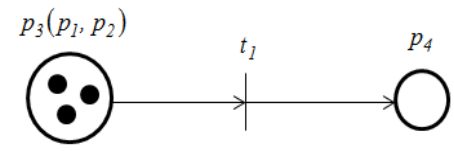

$\mathrm{b}$

Figure 1. Fragments of the model:

a - a model fragment constructed using a network (1)

$\mathrm{b}-\mathrm{a}$ model fragment constructed using a network (4)

The analysis of the above figures showed that the latter version greatly simplifies resource costs, therefore, the efficiency of CFPN will increase with increasing 
dimensionality of the model. This is especially true in distributed or hierarchical complex systems, which take into account, apart from objective and subjective factors, meteorological, geographic, physical and chemical. Most of these factors are poorly formalized, so their influence is realized on the basis of fuzzy expert assessments. The development of information technology has brought about significant changes regarding the mapping of the space of states of the interacting processes of complex systems, the integration of traditional approaches and geoinformatics has greatly influenced.

The tool for realizing the provisions of geoinformatics is geographic information systems that allow the use of modern object-oriented information technology and remote sensing technology for describing spatially distributed objects.

For research and modeling of specific spatial objects, models are used: digital spatial model of geospatial data, information model, mathematical model of the image. It should be noted that methods of geographic modeling of a complex geosystem and its components include modeling of structure, dynamics, interconnection, and also functioning of the system in space and time.

The main component of the simulation is the digital model of the terrain, which can be obtained using modern technology. The condition of perception of a digital map is the visualization of a cartographic image encoded on it by displaying its contents on the monitor screen.

In addition, distinguish the following types of information models: information descriptive, information resources, intellectual.

The information descriptive (descriptive) class includes models that are constructed as a description of a process, phenomenon or object, for example, a file, a text document. Information resource model is able to accumulate data for its improvement and optimization (database model). An intellectual model is capable of accumulation of information, self-improvement and implementation of actions based on knowledge-oriented technologies, the use of fuzzy logic, pattern recognition.

Information models are based on mathematical models of the image.

The problem of fuzzy spatial data modeling of complex spatially distributed objects is quite complex and multifaceted. The study suggests the creation of meteorological data based on applications of intellectual approaches that combine object-oriented databases and knowledge for modeling and queries of spatially distributed objects. The analysis of these works showed the importance and promise of research based on models that use fuzzy logic and knowledge-oriented technologies.

In the future, there are perspective studies based on the models of spatial analysis of the states of interacting processes of complex systems using fuzzy logic and knowledge-oriented technologies of geoinformatics.

\section{References:}

1. Tvoroshenko I. S. (2010). Analiz protsessov prinyatiya resheniy v intellektual'nykh sistemakh [Analysis of decision-making processes in intelligent systems]. Sistemy obrabotki informatsii, vyp. 2 (83), pp. 248-253. 
2. Kucherenko Je. I., Tvoroshenko I. S. (2011). Operatyvne ocinjuvannja prostoru staniv skladnykh rozpodilenykh ob'jektiv z vykorystannjam nechitkoji intervaljnoji loghiky [Operative evaluation of the space of states of complex distributed objects using fuzzy interval logic]. Shtuchnyj intelekt, № 3, pp. 382-387. 